\title{
Postdigital Interfaces and the Aesthetics of Recruitment
}

\author{
Darshana Jayemanne, Thomas Apperley, \& Bjorn Nansen
}

Transactions of the Digital Games Research Association

2016, Vol. 2, No. 3, pp. 145-172

ISSN 2328-9422

http://todigra.org

TEXT: Licensed under Creative Commons Attribution (CC BY-NC- ND

2.5) http://creativecommons.org/licenses/by-nc- nd/2.5/

IMAGES: All images appearing in this work are property of the respective copyright owners, and are not released into the Creative Commons. The respective owners reserve all rights.

\section{ABSTRACT}

This paper analyses reconfigurations of play in emergent digital materialities of game design. It extends recent work examining dimensions of hybridity in playful products by turning attention to interfaces, practices and spaces, rather than devices. We argue that the concept of hybrid play relies on predefining clear and distinct digital or material entities that then enter into hybrid situations. Drawing on concepts of the 'interface' and 'postdigital', we argue the distribution of computing devices creates difficulties for such presuppositions. Instead, we propose thinking these situations through an 'aesthetic of recruitment' that is able to accommo- 


\section{ToDiGRA}

date the intensive entanglements and inherent openness of both the social and technical in postdigital play.

\section{Keywords}

Hybrid games, interfaces, postdigital, practices, spaces, digital play, phenomenology, recruitment

\section{INTRODUCTION}

'Hybrid play' designates recent trends in product and game design that take advantage of new compositions of material and digital arrangements afforded by emerging technologies. These include micro-electronics, embedded sensors, and the 'app revolution', which are facilitated by a range of devices, infrastructures, protocols, and applications such as mobile devices, wireless networks, near-field communications (NFC) and tagging technologies such as RFID. Hybrid games include: locationbased or spatially-oriented forms such as 'pervasive', 'locative', 'augmented' and 'mixed' reality games (e.g. Montola 2011); as well as more technology or device-oriented forms such as computer-augmented board games, appcessory games and connected toys (e.g. Bergström and Björk 2014; Tyni, Kultima and Mäyrä 2013).

In turn, these game developments and associated idioms have challenged methods of analysing video games in terms of the classic arrangement of material devices (either console or PC-based) and virtual spaces. In many of these emerging games, the relations between embodied participation, mobile or sculptural devices and versatile digital elements call for the development of more theoretical and critical approaches to hybrid forms of gaming. Here, we argue that the contemporary situation of widely distributed computing complicates the device-focused 'hybrid dimensions’ approach developed by Tyni, Kultima and Mäyrä (2013) because we cannot pre-judge the spaces in which play will occur or the devices and bodies that will engage in ludic behaviour. As Berry and Dieter (2015) argue, the contemporary moment reflects a 'postdigital 
aesthetics' in which relations between space, time and bodies are 'in a regular state of constant upheaval'. We suggest it is productive to conceptualise hybrid gaming within the context of postdigital aesthetics - a situation in which it is increasingly difficult to pre-judge what will constitute the interface, and who, where and when different elements will be enrolled into such digital materialities of gaming. Thus, this paper offers some preliminary analysis towards conceptualising postdigital play, initially by identifying examples in terms of three scales of spatial practices: the conventional game space, the domestic space, and the public space. We then situate these examples within interface theories and criticism, and in particular the phenomenologically-informed elements of Farman's (2012) 'mobile interface theory', to argue for viewing hybrid play interfaces as an aesthetic of 'recruitment' which enrol and blur diverse spatial domains, social practices, and material lives.

\section{HYBRID DIMENSIONS: SYNCHRONY AND DEPENDENCE}

It is important to acknowledge that video games have always been 'playful hybrid products'. The recent passing of Ralph Baer enjoins us to recall his work and how it shows that the commonsense notion of what a video game 'is' or ought to be is a case of false concreteness: there are other possibilities and potentials for how games could have developed. Baer's work (along with technician Bill Harrison) led to the Magnavox Odyssey (1972), which was tied to the television and thus has some similarities with the contemporary game console, but Baer also experimented with peripheral input devices such as a light gun and visual elements such as translucent plastic screen overlays. The Odyssey shipped with traditional play aids such as tokens, score cards, chips, play money and game boards (Winter 2008, 50). Baer also worked on the electronic toy Simon (Milton Bradley, 1978) (Donovan 2010, 69-70), which is a precursor to some contemporary hybrid play products. Baer's bricolage resonates with those of other early creators such as William Higginbotham (creator of Tennis for Two), MIT's Tech Model Railway Club (inventors of Spacewar!), Will Crowther (creator of ADVENT) and many others. 
The history of video games, through arcade, console and PC generations, attests to a long tradition of creating intensive relations between a wide variety of designed objects, semiotic systems, forms of cultural capital, marketing techniques, and experiences of play. Video games are natively hybrid.

Current trends towards hybridity are thus something of a return to form. As in 2007 - when the Nintendo Wii showed up the narrowness of the industry's notions of unidirectional innovation by emphasising a novel control scheme rather than more powerful hardware - a renewed conceptual and scholarly emphasis on hybrid play is warranted today, as Mäyrä et al. (2014) argue in their introduction to a timely collection on the topic. The issue is all the more pressing because while the Wii's gestural controller re-deployed the relation between gamers and their domestic spaces, a survey of the contemporary gaming situation indicates that designs, techniques, objects, networks and bodies are proliferating and redistributing the digital materialities and relations of play across multiple hybrid dimensions - often challenging notions of public and private space.

Reconfigurations of material and digital elements, which are increasingly mobile, 'pervasive’, 'locative', ‘augmented' and 'mixed' (Montola 2011), can be characterised as postdigital in terms of gaming that is continuous with the digital, yet also exceeding the digital through conditions that are embodied, technical and historical (Berry 2014; Schinkel 2014). Broadly, then, the postdigital 'describes the messy state of media, arts and design after their digitisation...a media aesthetics which opposes such digital high-tech and high-fidelity cleanness' (Schinkel 2014), in which 'the historical distinction between the digital and the non-digital becomes increasingly blurred...[and] computation is part of the texture of life itself which can be walked around, touched, manipulated and interacted with in a number of ways and means' (Berry 2014, n.p.).

This complexity reveals difficulties with the 'hybrid dimensions model' of Tyni et al. (2013), which identifies two analytic dimensions: syn- 
chronicity and dependency. Synchronicity refers to the degree to which digital and material are experienced simultaneously in a given product (an app that can only be used in concert with a material toy has high synchronicity). Dependency indicates how integrated the elements are (a toy can be played with even if the app isn't loaded, so there is a low level of dependency). While this model is effective in characterising important differences between the games that Tyni et al. study such as Disney Infinity (Avalanche Software 2013), the Skylanders series (Toys for Bob 2011-) and Invizimals (Novarama 2009), the case studies are limited to commercial 'hybrid playful products' which assert highly determined regimes of production and consumption - they seem relatively clear and distinct both as designed objects and as games.

The concept of the postdigital challenges such binary logics because of the highly distributed state of contemporary computing technology. Where Tyni et al. tend to distinguish between a material toy and digital game space, for example, from a semiotic point of view the material toy is a signifier which can be analysed in terms of 'virtual' signifieds; similarly, the virtual space of the digital game has 'material' impacts on the bodies that engage with it (Farman 2012; Apperley and Jayemanne 2012). In the case of the 'connected toy', such as the controllable ball Sphero (Orbotix 2010), is whether or not the ball is working independently from the app the most interesting or pressing question for scholars to ask? In increasingly postdigital situations of play, there is clearly a need for more theoretically informed approaches.

Furthermore, by attending to well-defined commercial products, Tyni et al. tend to analyse the uses of each game from the point of view of 'proper' play that follows the clear design affordances of the game devices in question. Games are not always played as intended, and these forms of 'counterplay' are often considerably dynamised by people, events and objects in the locations where play takes place. As such, the focus on game technologies and their digital-material elements tends to leave aside questions about how such hybrid games may reconfigure the subjects or spaces in which they operate: for example, the complex 
domestic spatial ecology involving interrelations between family members, architectural layout, social media engagements, physical toys, digital screens, game consoles, wireless networks, and so on.

Instead of focusing on particular devices and therefore presupposing the contexts and spaces in which they are used, then, this paper explores the practices, materials and spaces that are opened up, occupied, or challenged by these hybrid games. Examples of hybrid play - which have seen game designs enter into new collaborative arrangements with disciplines such as urban design (de Souza e Silva and Sutko 2009), augmented board games (Bergström and Björk 2014), playground design (Poppe et al. 2014), and many more - go beyond the contexts of commercial product design, and suggest a need to account for the way that technologies, spaces, games and players enter into re-fashioned or unpredictable relations and thereby challenge designed uses and values.

These configurations of hybrid play considerably complicate the 'magic circle' model of play - so much so that the 'circle' can take on almost theological connotations (having its centre nowhere and circumference everywhere). If not precisely theological, for Farman (2012), the potential for pervasive games to make a magic circle appear in public and communal spaces has significant ethical dimensions. He examines geotagging gamers, whose behaviour can be confusing for onlookers. Players refer to non-players they encounter as 'muggles', a reference to the way characters in the Harry Potter series of books talk about people without wizardly abilities. Passers-by may be liable to become unwillingly 'immersed' in someone else's locative game. This leads Farman to raise the ethical question of how a game that takes place in public space should be played to minimise the involvement of unwitting or unwilling participants.

A contemporary example would be Google Glass, which was recently discontinued as a consumer technology (Warren 2015). The device caused discomfort for people who were concerned that they were being recorded without their permission (the term for Glass users changed from 
Google’s preferred 'explorers' to the vernacular 'glasshole'). Conceived as a kind of 'everyware’ (Greenfield 2006), Google Glass struggled in public space, but the company plans on re-releasing the device with an orientation to businesses (with an emphasis on healthcare and construction) - a second life in more contained and predictable contexts. Another example is the renewed interest in virtual reality headsets devices whose value putatively lies in the creation of a type of particularly bewitching magic circle - shipped with 'a nearly 1000-word set of “Gear VR Product Use Warnings”' (Orland 2014). Virtual reality, it seems, can’t help but keep crashing into reality. The 'magic circle', therefore, may be better reconceptualised as spatially, temporally and socially 'expanded' (Montola 2005) or perhaps as a set of 'frames' rather than a unitary bounded space (Mäyrä and Lankoski 2009; Jayemanne 2010).

Hybrid play thus takes place in contexts that are increasingly postdigital: their limits and characteristics cannot necessarily be predicted before they emerge in the process of play. Here we propose to modify and expand on the hybrid dimensions method of analysis from the point of view of diverse interface effects (Galloway 2012), rather than predefined devices or products. This theory informs the analysis of situations in which the set of playful actors, objects and spaces exceed discrete, pre-defined units (such as the 'hybrid playful products' Tyni et al. focus on) - accounting for a postdigital situation in which 'computation becomes experiential, spatial and materialised in its implementation, embedded within the environment and embodied, part of the texture of life itself but also upon and even within the body' (Berry and Dieter 2015, 3).

For Berry and Dieter, postdigital aesthetics involve the contemporary navigating of a complex field of distributed computing devices which entail a state of agnosis or 'not knowing'. This calls for a kind of 'agnotology':

By 'agnotology' we are referring to the way in which computation facilitates a systemic production and maintenance of ignorance. The tendency 


\section{ToDiGRA}

towards automated and accelerated modes of action complicates and may undermine structures of reflection and critique. One consequence is a twisting and turning of computational logics into other contexts against attempts to orient and 'get a grip' on computational things (Berry and Dieter 2015, $5)$.

In the context of hybrid play, the concept of the postdigital challenges assumptions that it is easy to know or readily identify what is digital/ virtual and what is physical/actual (and therefore whether they can be characterised as simultaneous or dependent) prior to analysing any given situation. In short, the hybrid dimensions model can be developed to account for multiplying hybridisations. One way to approach this problem is to theorise the abilities of interfaces to bring new bodies and spaces into relation - or, conversely, how an interface arises as the set of these abilities rather than as a predetermined 'thing'. Rather than conceiving the interface as a stable or determined object, it is understood as various practices of interfacing (Cramer and Fuller, 2008).

To achieve this, in the following sections we describe instances of hybrid play at three scales of postdigital spatial practice: the conventional game space (such as the PC or console setup or the board game), the domestic space, and finally the public space. In each case, we focus on the way that asymmetric and contingent bodies and spaces are organised by these interface effects. Following this, we draw on interface theories and criticism, and in particular Farman's (2012) phenomenologically-informed 'mobile interface theory' and concept of the 'sensory-inscribed body' as a way of approaching hybrid game play. The goal of this approach is not to analyse the synchronicity or dependency of a given object, game or product so much as to explore the post-digital synchronisations and dependencies at work in recruiting the playful objects and participatory subjects of interface play. This aesthetic of recruitment enrols increasingly diverse spatial domains, social practices, and material life.

\section{CONVENTIONAL GAMES, NEW HYBRIDITIES}

As has been noted above, while hybrid play is often presented as a newly 
emerging phenomenon, video games are already natively hybrid, postdigital constructs. However, various types of hybridisation may be more or less successful - even if they have considerable commercial power behind them. Microsoft's attempt to force the issue of hybridising the traditional functions of the console with always-on internet connectivity and Kinect motion tracking for their Xbox One console met with significant resistance. Introduction of new hybridities can also significantly alter the experience of play - for example, the introduction of voice communication can lead to very different MMO interactions than would otherwise be the case (Carter, Wadley and Gibbs 2012).

Video games are renowned for their ability to create immersive effects, but immersion is not limited to the attempt to recreate the 'unmediated' experience of phenomenal space - as many FPS games are supposed to do. It is possible to become as immersed in the third-person Dragon Age: Inquisition (Bioware 2014) or the godlike perspective of Civilisation $V$ (Firaxis Games 2010) as it is any FPS. In all such cases, the interface is highly effective in organising the proprioceptive, cognitive and other powers of a human player with the various capabilities of the devices that comprise the game apparatus. When video games work well they are excellent examples of what Farman describes as the sensory-inscribed body (a concept expanded below): an interface that players almost see 'through', looking as if through a window into another, virtual world.

However, as Consalvo (2007) has shown through the example of cheating, actual play often belies the magic circle thesis even in conventional video gaming - players often hybridise their experience of the game with 'paratexts' such as cheats, walkthroughs, FAQs and the like. The FPSMMO hybrid Destiny (Bungie 2014) saw players abandoning the typical mission structure to exploit a (subsequently patched) 'Loot Cave' where an infinite supply of weak enemies could be harvested for drops, and more recently adopt a method of beating a powerful raid boss by yanking the console's LAN cable (Schreier 2015). Even a successful effect of immersion may not endure with a particular player. Trying to play a once-loved game in an emulator can be an alienating experience: the 


\section{ToDiGRA}

interface's ability to organise the asymmetries between player and apparatus may not match up to a fond memory, or the control scheme may ruin the experience: in the failure of the magic circle, the hybridity of the game asserts itself against the illusionistic effect that was once so compelling.

New types of hybridity are also entering into traditionally 'bounded' gaming situations through internet connectivity. One example is the way that new games are hybridising in-game time with real-time elements. Dragon Age: Inquisition, for example, utilises a game mechanic called a 'War Table' in which they direct their subordinates to complete certain tasks. Regardless of whether the player logs in or out of the game, these tasks will be completed according to a real-world clock. The War Table thus opens up an interface effect between the game time and the rhythms of everyday life, providing an additional incentive to return to the game at regular real-time intervals.

A more comprehensive utilisation of an interface between game time and everyday life can be found in Destiny, which staggers its missions across real time periods (some can be done at will, some once a day, others weekly). While this is a structure that is common to many MMORPG games, Destiny is primarily a FPS: the requirement for internet connectivity in current generation consoles has facilitated the appearance of real-time structures in other genres:

Each day in Destiny resets at 1AM Pacific, 4AM Eastern. At Tuesday's reset, the new week also kicks over, meaning that your weekly mark cap resets, the weekly strike changes, and your raid progress and drops both reset (Hamilton 2015).

Another increasingly apparent hybridisation that is affecting conventional video games derives from the capacity to easily stream gameplay to the internet via sites such as Twitch.tv. A genre has formed around live play in which players specifically tailor their performances to be disseminated across YouTube or Twitch.tv channels. Often this will also involve interaction with viewers via the integrated chat stream (a prac- 
tice prevalent enough that Twitch.tv has developed its own emoji idiom). A new element is added to the process of embodied spatiality of the game: one oriented to an interface with a distributed public. Many live streamers attest that exhibiting their performance in this way places new cognitive and performative loads on their embodied interaction with the game apparatus. This can therefore be classed as a new type of hybrid play in its own right. Popular livestreams are often made by expert players for the purpose of disseminating effective styles of play, an element that complicates Consalvo's (2007) notion of gaming 'paratexts'. Here, the paratext is not epiphenomenal: it is woven simultaneously as a new hybrid performance of a given game.

Video sharing gameplay is not the sole preserve of expert players. In fact, the most highly viewed YouTuber as of 2014, Felix Kjellberg or 'PewDiePie', has made his mark by failing in an entertaining fashion. Utilising a webcam setup so that viewers can see his reactions in an inset window, Kjellberg's most famous performances are of horror games such as Amnesia: The Dark Descent. In a powerful example of Farman's concept of how embodied spatiality is distributed through technological media, Kjellberg's audience jumps and screams along with his image projected across the livestream or in an archived video. Although distributed across the world, the hybrid play of the video stream is an interface that lets viewers participate in the horror game's intensive processes of generating spatial experience through the sensory-inscribed body. Another livestream of Amnesia: The Dark Descent, made by Starcraft 2 commentator Sean 'Day9' Plott, saw Plott's friends send him text messages during the stream. Already terrified by the game, Plott's commentary expands from what is evidently a highly immersive game world to involuntarily hybridise this new device: “I don’t know why I asked my friends to text me... my phone went 'bzz' and it frightened me. I just have to mute this so it doesn't freak me out so much.” Slightly later in the stream, a text message causes Plott's phone to vibrate during a particularly harrowing gameplay moment, "Gah, stop it! Stop texting me! Who's watching? Friends! Don’t text me! Leave me alone!” Here the intensive processes by which the horror game entangles physiological 
and perceptual human capacities with various computer-generated stimuli are also distributed across the networked public of the stream, and the personal network defined by access to an individual's mobile phone number.

\section{POSTDIGITAL PLAY IN DOMESTIC SPACES}

Whilst Tyni et al. treat the domestic space as a relatively neutral background in their analysis of hybrid playful products such as the Skylanders series, game studies could benefit from an engagement with research traditions such as technology domestication and parental mediation (Nansen and Jayemanne, forthcoming). These fields both add considerable complexity to the scholarly study of home life and the relations between family members, particularly in terms of children's access to technological devices. The rhythms of everyday life (Apperley 2010) thus exercise a powerful influence over hybrid play in the domestic space.

The advent of crowdfunding platforms such as Kickstarter has given impetus to many playful products that operate at intermediary economies of scale. While they are unique devices, many of these products rely on technologies that have already been subject to the process of domestication - such as tablets and mobile phones, which are often themselves hybrid ensembles of devices such as cameras, gyroscopes and so on in order to operate. An example of this is Osmo, a crowdfunded augmented reality product that hybridises an iPad, a stand, a reflector that directs the device's camera towards the surface that it stands on, an app for download and physical game pieces such as a classic tangram. The app dynamically reacts to activities undertaken on the physical surface through several games, effectively turning the surface (typically, a table) into an interface.

Osmo's marketing video shows an adult in a pure white room setting up the tablet for play on a table. A child emerges out of this idealised domestic space and begins to manipulate the tangram pieces to create 
shapes and perform other activities. This draws another child, and then more, until a crowd has formed. While this is a typically decontextualised advertising situation (nobody starts crying because they stepped on a tangram piece, for example), the robust feedback between the children's activities as they collect objects such as a toy dinosaur to place before the device and witness its response suggests a space in which many different processes of embodied interaction can be explored.

The operative aesthetic of childlike fascination and proliferating possibility is common to many of these devices. The intensive potentials for play exhibited by devices such as Osmo represent an aspect of postdigital gaming that we term an 'aesthetic of recruitment'. That is, an enrolment of player bodies and sensations that moves beyond the screen, as well as an enrolment of many different material and digital elements that may be distributed through the postdigital domestic space. This is a key selling point of the device, as attested by the way the video depicts both children and many varied objects coming together to configure Osmo's different interface effects and processes of sensory-inscription. Cameras and other types of sensors, when combined with appropriate apps, diversify the number and types of bodies that can be gathered within and through an expanded range of sensory-inscriptions in a given play situation. In turn, the apparent 'contagiousness' or fascination of playful behaviour encouraged by these devices informs the affective aesthetics of recruitment.

As noted, however, the sterile background of the Osmo advertisement does betray the fact that the system and its recruiting powers are curated for a very particular hybrid device: the advertorial camera. The aesthetic of recruitment is taken further still by MaKey MaKey, a circuit board which allows players to 'make anything into a key' by attaching alligator clips to objects. This then enables the objects to work as keys for inputting commands into a computer, opening up the objects of domestic space and beyond - bananas to stairs to tubs of water and even pets to become recruited as new types of interface within a vastly expanded postdigital and material repertoire of hybrid play. MaKey MaKey adver- 
tisements tend to take place in a far more cluttered mise-en-scene than those of Osmo, reflecting the wider gamut of objects and player behaviours which it can draw into a hybrid system. The digital elements are, however, less diverse: generally, the technology seems to have been used to control existing games such as PacMan or Super Mario Bros, but the physical and tactile experience of play is considerably transformed by the introduction of highly unorthodox materials into the interface. The aesthetic of recruitment thus expands the possibilities for new proprioceptive relations to game-space by forcing players to re-balance, re-configure and re-consider their habitual use of a versatile interface effect.

Osmo and MaKey MaKey represent two different styles or 'grammars' of postdigital recruitment. Osmo draws players inwards to a relatively bounded space to play with a set of pre-made physical objects that serve specifically designed digital games. MaKey MaKey (at least as advertised) pushes a pre-existing virtual game world outwards to hybridise with the environment and objects in new ways. The potentials for asymmetrical recruitment offered by the latter are greater than that of Osmo simply because of the sheer amount of objects that are available for recruitment, as well as the way to activate the powers or virtues of such bodies (a control scheme made of bananas 'feels' very different than one made of play dough). Despite the contrast of inward and outward recruitment emphasised by either Osmo or MaKey MaKey, in both cases we see the assembly of asymmetric sets of sensory-inscribed bodies - less a predefined interface than various sensory practices and digital materialities of interfacing.

\section{MOBILISING POSTDIGITAL PLAY IN PUBLIC SPACES}

Sphero is a 'connected toy' consisting of a mechanised ball that can be rolled around by a phone app. In the 'Sphero 2.0' advertising spot, a rapid montage of the device rolling extends beyond domestic space and engages small groups of people to also move through public locales such as parks, beaches, city squares and so on. The marketing moves seamlessly from context to context in a world where everyone seems happy 
to see it and be recruited in the fun. In reality, however, Sphero isn't really appropriate for many of the spaces it is shown moving through, on a train platform or the city square, where it is close to moving vehicles and weaving under the feet of passers-by. The public spaces Sphero is shown moving through have little bearing on the everyday uses of connected toys, rather they suggest and aestheticise the recruitment of 'the public'.

Where recruiting players and objects within the domestic space has its own set of concerns and issues, ethical concerns are more clearly pressing in public space. Issues of privacy and consent become critical because not everyone will want to be recruited into a particular game, and even if they do, ensuring that they understand the implications of their recruitment becomes a significant issue. This is evident in what was eventually an un-funded crowd funding campaign smartphone game, which wirelessly connects to a rubber grip with inbuilt sensors, called Hybrid Play. The setup had the explicit aim of transforming playgrounds into video games by attaching the sensor clip to play equipment, such that playing on the physical equipment controls digital game elements. The enrolment of children's outdoor play spaces and playground equipment raises ethical questions about the kinds of participants and audiences recruited in hybrid play arrangements, and in particular the potential for digital 'corruption' of traditionally physical materials and active interfaces.

However creating mobile interfaces in public space also creates opportunities for new scales and types of play such as locative, mixed-reality and pervasive games. Scale plays an important role in how these games operate. Large corporations such as Google's Niantic Laboratories have created location-based and mixed-reality games like Ingress (2012) and Pokémon Go (2016) that recruit large swathes of urban space. Ingress, for example, separates players into two factions who have varying philosophical approaches to an influx of 'exotic matter' and 'anomalies' into our world. Players utilise their mobile phones, along with an augmented 
reality app, to locate and capture points in space, and by linking these points can establish areas of control (see Moore 2016).

The design of Ingress seeks to minimise the potential for players to overtly disturb or 'recruit' the people around them in two main ways. Firstly, the game's fiction suggests that public sculpture is particularly prone to anomalies. This means that players are unlikely to stray into private property during their activities, and that the points are more likely to be located in highly-trafficked regions that will help players incorporate their gaming into everyday movements. Secondly, the 'in-game' actions are contained within the mobile phone device. When at a location, the activity of the players will not appear too different from a normal mobile phone user taking and manipulating a photo or video. Ingress thus carefully tunes the bodies of its participants such that their sensory capacities are magnified (through the augmented-reality app) while their culturallyinscribed behaviours are restrained - although this is not always successful, as loitering, repetitive movement and so on may still be perceived as suspicious in their own right (PiedType 2013).

This type of surreptitious public play can be contrasted with games in which postdigital public space and digital technologies interact in a more overt fashion, such as public games of Johann Sebastian Joust. Here the problem of recruitment is modulated by the clear 'playful' behaviour of the participants (moving their bodies and controllers in a bounded space), and connects with traditions of street art and theatre. One interesting space which highlights the role of the sensory-inscribed body and the process of recruitment, has involved the use of public transport tram networks in a locative game in which the location itself is mobile. The movement of the vehicle affects the physical design requirements of the game, while the movement of passengers on and off the tram means that recruitment has to be versatile. 'Cart-Load-O-Fun', a game designed for the tram system in Melbourne, Australia by researchers at RMIT University, took these hybrid dimensions into account by using pressure pads to make controllers out of the vehicle's hand rails (Toprak 2013). Passengers could then partake in a game which allowed them to maintain 
their balance in response to the acceleration and deceleration of the tram, while squeezing the pads and playing a digital game.

\section{DISSCUSSION: INTERFACE THEORY AND POSTDIGITAL PLAY}

We turn to theorising the aesthetic of recruitment installed in the above examples of postdigital interfaces by drawing on critical interface studies, and in particular Farman's 'mobile interface theory'. The concept of a mobile interface as suggested by Farman (2012) may seem like an odd one given that the common computing interface metaphor of a 'window' gives the image of a static mediating boundary between two spaces (one real and one virtual). In this metaphorical understanding, the interface is a facilitator of movement rather than being mobile in itself. The idealised 'user-friendly' interface almost disappears entirely, giving smooth and uncomplicated access from the world of flesh-and-blood users to immense vistas of digital content - as Bolter and Grusin put it, 'What designers often say they want is an 'interfaceless' interface... the user will move through the space interacting with the objects 'naturally,' as she does in the physical world' (2000, 23). The seeming straightforwardness of this conception has, however, been challenged by many scholars and designers, including Drucker (2011), who critically engages with interface theory to challenge the concept of the interface as a fixed thing rather than a performative relation:

Interface is a dynamic space, a zone in which reading takes place. We do not look through it (in spite of the overwhelming force of the 'windows' metaphor) or past it. The desktop metaphor at least suggests a space of activity in which icons stand for objects with behaviours we enact...If we usually separate what we think of as 'content' from the wireframes and display techniques, then we are performing acts of blindness (Drucker 2011, 9).

For Drucker, the desktop metaphor is superior to that of the window because it involves the manipulation and mobility of multiple objects across a dynamic surface, rather than the scopophilic 'view through a 
window'. The interface concept has a broader remit than the digital technologies it is typically applied to:

We do not read content independent of interface on a screen any more than we do when we read the newspaper. We have only to strip away the graphical codes of a printed text - put its letters and words into a simple sequence, remove paragraphing, hierarchies, word spacing etc. - to see how dependent we are on these format elements as an integral part of meaning production' (Drucker 2011, 9).

Two major points arise from Drucker's discussion, both of which we have flagged in our examples. First, it is important to register the asymmetry of the spatial forms organised by interfaces: the metaphor of the transparent window should give way to that of the cluttered desktop. Where the spaces on either side of the window share an equivalent status, the hybrid space of the desktop is one characterised by the different powers, capacities and limitations of the objects that constitute it. Mediated space is thus not conceptualised as an empty opening in which things happen to take place; instead, it is produced in a set of operations that resemble Goffman's 'frame analysis' of social distinctions and the resulting 'organisation of experience' (1974). This is a point also emphasised by Cramer and Fuller (2008), who trace the historical usage of the term 'interface' to chemistry: 'Interfaces describe, hide and condition the asymmetry between the elements conjoined. The asymmetry of the powers of these bodies is what draws the elements together' (150).

Second, because interfaces are often useful to the degree that they are 'designed to dissimulate their function as interfaces' (Cramer and Fuller 2008, 152), they are often most apparent and legible at moments of contingency, such as when the functionality of a previously stable interfacial relation breaks down - we may take the complex operations of an ingame camera for granted until it bugs out at a crucial gameplay moment, for example. Reading is structured in graphical codes that 'perform a quasi-semantic function, not merely a formal or syntactic one' (Drucker 2011, 9). Stripping the expected conventions from the written word serves to make a message illegible, even if it is otherwise the exact same 
sequence of signs. This failure exhibits the contingency of the codes that govern communications systems: broken down or taken out of context, they show themselves to be less universal and more prone to hybridity than we may habitually acknowledge. Here, Galloway's notion (2012) of hybridity as registered through various 'interface effects' construes interfaces as processes rather than objects of media. That is, instead of seeing an interface as a surface or media device, such as a screen or keyboard, moments of failure render visible their effects of mediation on the operation of sensory, cultural and material practices.

If the desktop has certain advantages over the window as a metaphor for the hybridity and diversity of interface operations, it may be less apt with regard to an increasingly postdigital world. Indeed, as Farman points out, one of the pioneers of ubiquitous computing ('ubicomp'), Mark Weiser, wrote an influential piece in 1993 called 'The world is not a desktop'. While Weiser overtly makes the case for 'invisible' interfaces and computing, what is of more interest in this context is the way he goes about doing so: that is, by expanding the set of metaphors that we use when we think of how interfaces operate. In particular, Weiser criticises the tendency to imagine interfaces as replicating contemporary conscious forms and modalities of behaviour. Thus where the multimedia of the time sought to emulate the experience of legacy media such as TV, virtual reality sought to emulate unmediated experience, and intelligent agents sought to replicate human agents, Weiser suggests a certain, almost dialectical, relation between visible and invisible technology (and hence, interfaces between technologies and bodies):

Invisible technology needs a metaphor that reminds us of the value of invisibility, but does not make it visible. I propose childhood: playful, a building of foundations, constant learning, a bit mysterious and quickly forgotten by adults. (Weiser 1993, online)

Weiser's nomination of childhood as a metaphor for interfaces means that he has a claim to being a forerunner of thinking on ubiquitous digital play in addition to ubiquitous computing - or, at least, as a proposal of an intimate relation between the two fields of possibility. This is certainly 
an aesthetic that MaKey MaKey and Osmo try to recruit. His description of the fertile and energetic potentials of ubiquitous computing resembles Caillois' (2006) notion of the freeform paidea more than the rulebound ludus. The world is no more desktop (or for that matter, console) than magic circle. Digital play expands throughout the street, the home, the park, the workplace and beyond. Importantly, Weiser's metaphor of childhood speaks to processes both of constant learning (the negotiation and re-negotiation of asymmetric relations) and forgetting (entropy, failure and error). The potentials of diverse and pervasive playful computing shift the critical goal from analysing this or that bounded play situation, towards tracing and navigating the emergence of what Fuller calls 'seaminess' (2007) across which the asymmetrical powers of bodies for sensing, feeling and doing are enrolled and hybridised in postdigital situations.

\section{PLAY AND AN AESTHETICS OF RECRUITMENT}

The postdigital calls for a conception of play that is capable of engaging bodies and flows that we cannot necessarily predict without arbitrarily reducing either the asymmetry or the contingency of the play situation the very mobility of many of the devices crucial to hybrid play designs means that it is not possible to definitively pre-judge their context of use. The boundary of the play space must be left an open question. Adding to work by game studies scholars such as Mäyrä, Tyni and Montola, as well as the tradition of HCI scholars who have focused on embodiment since Winograd and Flores (1986) and Dourish (2001), here we propose to draw on Farman's 'mobile interface theory' (2012). This was developed to trace the locative and pervasive effects that arise in the use of devices such as mobile phones, and questions of navigation and the orientation of embodied experience are highlighted. Farman combines two major streams of thought - phenomenology and poststructuralism, with key references in Merleau-Ponty (1958) and Derrida (1998) respectively, in order to produce a theory of the 'sensory-inscribed body'. In this con- 
text we will place the emphasis on the phenomenological aspects of the theory.

Phenomenology facilitates a focus on the integral relation between space, practice and body: 'embodiment is always a spatial practice... Bodies always take up space and, as Lefebvre argued, are spatial in and of themselves' (Farman 2012, 19). A particularly important nuance of this claim is that 'embodiment does not always need to be located in physical space. As people connect across networks on a global level, what many are experiencing as they practise the space of the network is embodiment... we create our bodies across digital media' (22). This creation involves the adoption of determinate spatial practices in which the interface - pace Drucker and Weiser - is liable to fade in and out of visibility. During a mobile phone call:

The interface of the phone typically recedes and you are moved into the space of conversation. If, however, there becomes an extended period of silence, the sense perceptions immediately pull focus from the other person to the device... You will move the phone away from your ear to look at the screen, determining if you are still connected, if your reception is strong, or if your battery has died (Farman 2012, 28).

The shift between the co-location of interlocutors to the surface of the phone screen constitutes two kinds of embodied spatiality; two distinct 'interface effects' (Galloway 2012). Farman also gives a more complicated example, which will be familiar to many scholars, of a student's phone ringing during a lecture. The confused behaviour of the individual student, the lecturer and the rest of the class show different processes of embodied spatiality, which usually flow smoothly, brought into focus at their moment of failure.

Farman argues that these situations indicate a need to reconsider the seemingly simple distinction between the virtual and the real, 'For, if it were an accurate opposition, then that which is virtual would also be considered "not real"' (2012, 22) - whereas the 'virtual' interface and digital content do perfectly real things: the pairing 'virtual/actual' is more helpful. He traces the concept of the virtual back to the term 'virtue', 
as it was used until the late 1400s, in the sense of the 'virtues' or powers and abilities of a given thing. However, it is not always the case that all the virtues of an object are actualised at once (as in the case of the mobile phone in the lecture theatre, where multiple possibilities for behaviour are urgently possible at the one embarrassing moment). Where the virtual/real opposition is a symmetrical relation in which the virtual 'mirrors' the real, the virtual/actual opposition is 'asymmetrical' both because the set of virtual possibilities tends to exceed any actual result, and because every process of actualisation is different.

This virtual/actual pairing informs another crucial notion that Farman derives from phenomenology: the importance of the habituated aspects of our sensory and embodied experience. This once again speaks to the processes by which interfaces come into and recede from 'visibility' (although it might better be termed 'perceptibility' because this refers to all the senses and not just sight). 'While those things that we are aware of and perceive are vital to our sense of being-in-the-world, our senses also work to block out much of the sensory input that we are bombarded with...Imagine that while you were having a conversation with someone, that every other conversation in the room and every sound in the room became equally important... We function as embodied beings because we do not notice everything or sense everything' (27). The senses are not simply receptive, but actively screen and sort phenomena in the process of producing spatio-embodied beings. Phenomenology terms this process 'proprioception'. At the same time, this sense of ourselves is inscribed by 'cultural inscriptions of masculinity or femininity, the signifiers of our cultures, or sexualities, our religions, among other aspects of our embodied identity that we read in others and encode on our bodies for others to read' (32).

Just as with our awareness of other subjects, objects can enter and become part of the process of proprioception that informs the sensoryinscribed body and its relations to foreground or background space. Where Merleau-Ponty uses the example of a person wearing a hat who instinctively ducks to clear a doorway without knocking it off, Farman 
extends the logic to technological objects such as vehicles and locative media devices. We argue that this approach can also be fruitful in the context of doing game studies in the era of postdigital play by providing a means of analysing how sensory-inscribed bodies arise in asymmetrical and contingent play situations - an aesthetics of recruitment in gaming configurations.

Rather than an assumed situation in which elements are symmetrical (at least to the extent that they can mutually enter into hybrid relations of digital and material, dependence and independence) under ideal conditions (a magic circle of prescribed behaviours and neutral space in which new and potential hybridisations are left unregistered), tracing postdigital play interfaces enables us to account for how sensory-inscribed bodies with asymmetric powers cohere and dissipate in playful spaces, sensations and practices. The acknowledgement of asymmetrical powers in postdigital play is one way of responding to the agnotology called for by Berry and Dieter (2015).

The postdigital interface helps us to analyse forms of hybrid play - in each case, we can pose questions about how spatial forms (locative, pervasive, bounded) are produced in relation to what types of sensoryinscribed bodies. This allows us to place the 'panoply of devices' foreseen by Weiser into relation with players' sensuous and cultural capabilities. Postdigital games (keeping in mind that video games are, at least to a degree, natively postdigital) enjoin us to take account of how multiple performances and spatialities are produced by the ensemble of framing devices at work in a given gaming situation. The magic circle as outward bound, should it be observed in a particular case, is produced by the integral operations of the various participants in playful processes: multiple synchronisations and de-synchronisations, multiple processes which result in various degrees of dependency and autonomy. Any one of these processes may exceed any given object or product and hence, it potentially troubles any analysis which relies on such a category because portable products can wind up in many different contexts that exceed their design parameters. We have argued instead for view- 
ing postdigital play interfaces as an aesthetic of recruitment, in which an analysis of the asymmetry and contingency of hybridity emerges through attention to the various spatial domains, social practices, sensory experiences, and material actors that are enrolled into, and distributed across, assemblages of play.

\section{CONCLUSION}

Engaging with critical interface studies and phenomenological theories of embodied action provides us with useful frameworks with which to study the burgeoning field of postdigital play. Moving beyond the metaphor of the window or the desktop towards the 'panoply of devices' imagined by Weiser, the interface approach seeks to account for how the asymmetrical powers of various bodies are organised in a given play situation - effectively, expanding the hybrid dimensions that we can analyse. This is particularly useful in the study of locative, pervasive and mixed-reality games because it helps us articulate how sensory-inscribed bodies and the hybridisations of play are co-generated in each case.

Drawing on concepts of the 'interface' and 'postdigital', our discussion has identified new hybridities in conventional games that are facilitating the emergence of new practices of play (as well as reaffirming the inherent hybridity of video games). It has also explored the interface effects that are being created in domestic and public spaces, which operate to create new relations between digital and material elements. Organised less around the interface as a determined object than around spaces, bodies, and practices of interfacing with increasingly undetermined digital materialities, we have proposed the term an 'aesthetic of recruitment'.

\section{Acknowledgements}

This research was supported through funding from an Australian Research Council (ARC) Discovery Early Career Researcher Award (DE130100735) and (ARC) Discovery Project (DP140101503). 


\section{References}

Apperley, T. Gaming Rhythms: Play and Counterplay from the Situated to the Global. Institute of Networked Cultures, Amsterdam, 2010.

Apperley, T. and Jayemanne, D. “Game Studies’ Material Turn”, Westminster Papers in Communication and Culture, 9.1 (2012), pp. 7-25.

Bergström, K. and Björk, S. "The Case for Computer Augmented Games” in ToDiGRA 1,3. Accessed 02/01/2015. Available at: http://todigra.org/index.php/todigra/article/view/32/49

Berry, D. The Post-Digital Ornament, Stunlaw blog post, 06 |June, 2014. Accessed 02/01/2015. Available at: http://stunlaw.blogspot.com.au/ 2014/06/the-post-digital-ornament.html.

Berry, D. and Dieter, M. (eds.). Postdigital Aesthetics: Art, Computation and Design. Palgrave Macmillan, London and New York, 2015.

Bolter, J. and Grusin, R. Remediation: Understanding New Media. Cambridge, MA., The MIT Press, 2006.

Bonsignore, E., Hansen, D., Toups, Z., Nacke, L., Salter, A., Lutters, W. "Mixed Reality Games" in Proceedings of the ACM 2012 Conference on Computer Supported Cooperative Work Companion (New York, NY, 2012), pp. 7-8.

Caillois, R. "The Definition of Play and the Classification of Games,” in The Game Design Reader: A Rules of Play Anthology. The MIT Press, Cambridge MA, 2006.

Consalvo, M. “There is No Magic Circle,” Games and Culture 4.4 (2009), pp. 408-417.

Cramer, F., and Fuller, M. "Interface,” in Fuller, M. (ed.) Software Studies: A Lexicon (149-152). The MIT Press, Cambridge, Mass., 2008. 
Derrida, J. Of Grammatology. Translated by G. Spivak. The Johns Hopkins University Press, Baltimore MD, 1998.

De Souza e Silva, A., Sutko, D.M. "Playing Life and Living Play: How Hybrid Reality Games Reframe Space, Play and the Ordinary” in Critical Studies in Media Communication, 25.5 (2008), pp. 447-465.

Donovan, T. Replay: The History of Videogames. Yellow Ant, Lewes, 2010.

Dourish, P. Where the Action Is: The Foundations of Embodied Interaction. The MIT Press, Cambridge, Mass., 2001.

Drucker, J. "Humanities Approaches to Interface Theory" in Culture Machine 12 (2011), pp. 1-20.

Farman, J. Mobile Interface Theory: Embodied Space and Locative Media. Routledge, London, 2012.

Fuller, M. Media Ecologies: Materialist Energies in Art and Technoculture. The MIT Press, Cambridge, Mass., 2007.

Galloway, A. The Interface Effect. Polity, Cambridge, 2012.

Goffman, E. Frame Analysis: An Essay on the Organisation of Experience. Harper and Rowe, London UK, 1994.

Greenfield, A. Everyware: The Dawning Age of Ubiquitous Computing. New Riders Publishing, San Fransisco CA, 2006.

Jayemanne, D. "Magic Frames: The Best of All Possible Virtual Worlds” in fibreculture 16, 2010. Accessed 14/04/2015. Available at: http://sixteen.fibreculturejournal.org/magic-frames-the-best-of-all-possible-virtual-worlds/

Mäyrä, F., Heljakka, K., and Seisto, A. “Introduction”, Transactions of the Digital Games Research Association 1,3 (2014). 
Mäyrä, F. and Lankoski, P. "Play in Hybrid Reality: Alternative Approaches to Game Design” in De Souza e Silva and Sutko (eds.). Digital Cityscapes: Merging Digital and Urban Playspaces (129-147). Peter Lang Publishers, New York, 2009.

Merleau-Ponty, M. The Phenomenology of Perception. Translated by Colin Smith. Routledge Press, New York NY, 1958.

Montola, M. “A Ludological View on the Pervasive Mixed-Reality Game Research Paradigm,” Personal and Ubiquitous Computing 15 (2011), pp. 3-12.

Moore, K. (2016). Sort Mii Out: Learning to Value Portable Gaming Encounters Through Nintendo's Streetpass Software. Games and Culture. Online first doi:10.1177/1555412016635814

Nansen, B., and Jayamene, D. "Infants, Interfaces, and Intermediation: Digital Parenting in the Production of 'iPad Baby' YouTube Videos. Journal of Broadcasting and Electronic Media (forthcoming).

Orland, K. "Samsung shares horrifying laundry list of potential Gear VR risks.” Accessed 20/01/2015. Available at: http://arstechnica.com/gaming/2014/12/samsung-shares-horrifying-laundry-list-of-potential-gearvr-risks/

PiedType. “Grandma Playing Ingress Stopped By Cops.” Accessed 10/ 01/2015. Available at: http://piedtype.com/2013/06/29/grandma-playing-ingress-stopped-by-cops/

Poppe, R., van Delden, R., Moreno, A., Reidsma, D. "Interactive Playgrounds for Children” in A. Nijholt (ed.), Playful User Interfaces: Gaming Media and Social Effects (99-118). Springer Science \& Business Media, Singapore, 2014.

Richardson, I. "Faces, Interfaces, Screens: Relational Ontologies of Framing, Attention and Distraction”, in Transformations 18 (2010), 
172 ToDiGRA

http://www.transformationsjournal.org/journal/issue_18/article_05.shtml.

Schinkel, W. What is 'Post-digital'? Rsearch tumblr, 09 July, 2014. Accessed 02/01/2015. Available at: http://rsearch.tumblr.com/.

Schreier, J. "Destiny's Latest Exploit: Pulling out Your LAN Cable”, Kotaku. Available at http://kotaku.com/destinys-latest-exploit-pullingout-your-lan-cable-1677068155.

Toprak, C., Platt, J., Ho, H., Mueller, F. “Designing Digital Games for Trams," in Proceedings of FDG 2013.

Tyni, H., Kultima, A., and Mäyrä, F. "Dimensions of Hybrid in Playful Products,” in Proceedings of AcademicMindTrek '13 (New York NY, 2013), ACM Press, pp. 237-244.

Tyni, H. and Sotamaa, O. "Material Culture and Angry Birds," in Proceedings of Nordic DiGRA (2014).

Warren, C. "First Version of Google Glass Discontinued". Accessed 20/01/2015. Available at: http://mashable.com/2015/01/15/google-endstony-faldell-nest/

Weiser, M. “The World is Not a Desktop” in Interactions (1994), pp. 7-8.

Winograd, T. and Flores, F. Understanding Computers and Cognition: A New Foundation for Design. Addison-Wesley Professional, Norwood, NJ., 1986.

Winter, D. “System profile: The Magnavox Odyssey,” in M. J. P. Wolf (ed.) The Video Game Explosion: A History from Pong to Playstation ${ }^{\circledR}$ and Beyond (50-51). Westport, Greenwood Press, 2008. 